\title{
GAMMA-RAY SPECTROMETRY OF GALACTIC SOURCES IN THE ENERGY RANGE 0.2-3.0 MeV
}

\author{
A. BUI-VAN, G. VEDRENNE, and P. MANDROU \\ Centre d'Étude Spatiale des Rayonnements, Toulouse, France
}

\begin{abstract}
A low energy gamma-ray telescope $(0.2-3 \mathrm{MeV})$ has been studied in our laboratory. It is made up of an active honeycomb collimator of $\mathrm{NaI}(\mathrm{Tl})$, with a geometrical angular aperture of 1.5 , and of a detector composed of $73 \mathrm{NaI}(\mathrm{Tl})$ sticks $(0.5 \mathrm{~cm}$ diam. $-5 \mathrm{~cm}$ length) incorporated in a plastic gabarit used in anticoincidence.

The directivity of the telescope is obtained at low energy by the collimator, and at higher energy by the directivity which exists in the gamma-ray interaction through the Compton and photoelectric effects. The electrons emitted in these interactions which are not contained in the sticks of $\mathrm{Na}$ ( $(\mathrm{Tl})$ are detected by the plastic anticoincidence scintillator. Only laboratory tests are presented here, for the study of the galactic gamma-rays, however, the system must subsequently be protected by a $4 \mathrm{~cm}$ thick anticoincidence well. In fact the detector directivity enables a great increase in the signal to noise ratio to be expected without greatly increasing the thickness of the shielding.

According to the results obtained the estimation of the minimum exposure time for the " $\mathrm{Crab}$ Nebula' would be about $2^{\mathrm{h}} 30^{\mathrm{m}}$ to have a signal at $1 \mathrm{MeV}$ with $2 \sigma$ of confidence above the background.
\end{abstract}

\section{Introduction}

The gamma-ray flux which continually reaches the balloon instrumentation causes an important background in the detector. Thus at the altitude of $3.5 \mathrm{~g} / \mathrm{cm}^{2}$ and in the energy range $0.1-3 \mathrm{MeV}$, atmospheric and cosmic gamma rays can produce a counting rate of about 7 photons $/ \mathrm{cm}^{2} \mathrm{~s}$ (Cline, 1961; Metzger, 1964). While galactic sources such as Crab Nebula must be about $\frac{1}{10}$, weaker.

As the expected gamma-ray sources have very low apparent diameters the measurement of their intensity depends on the detection area. Therefore an efficient method for increasing the signal to noise ratio is based on the utilisation of detector with a high angular resolution.

The proposed detector associated with an active honeycomb collimator has been studied in order to detect gamma-ray galactic sources. This paper gives the calibrations obtained in the laboratory. These first results show the angular resolution which can be obtained at different energies and consequently the possibility of reducing the shielding surrounding the detector.

\section{Technical Investigations}

\section{A. HONEYCOMB COLLIMATOR STUDY}

The main difficulties in building an active collimator are mechanical stress. The fragility of the crystal, limits the dimensions of the collimator holes, but the minimum geometric aperture of the collimator which can be used for experiments in space depends only on the efficiency of the telescope shielding against a gamma-ray background. Indeed this efficiency and consequently the weight of the shielding must be 


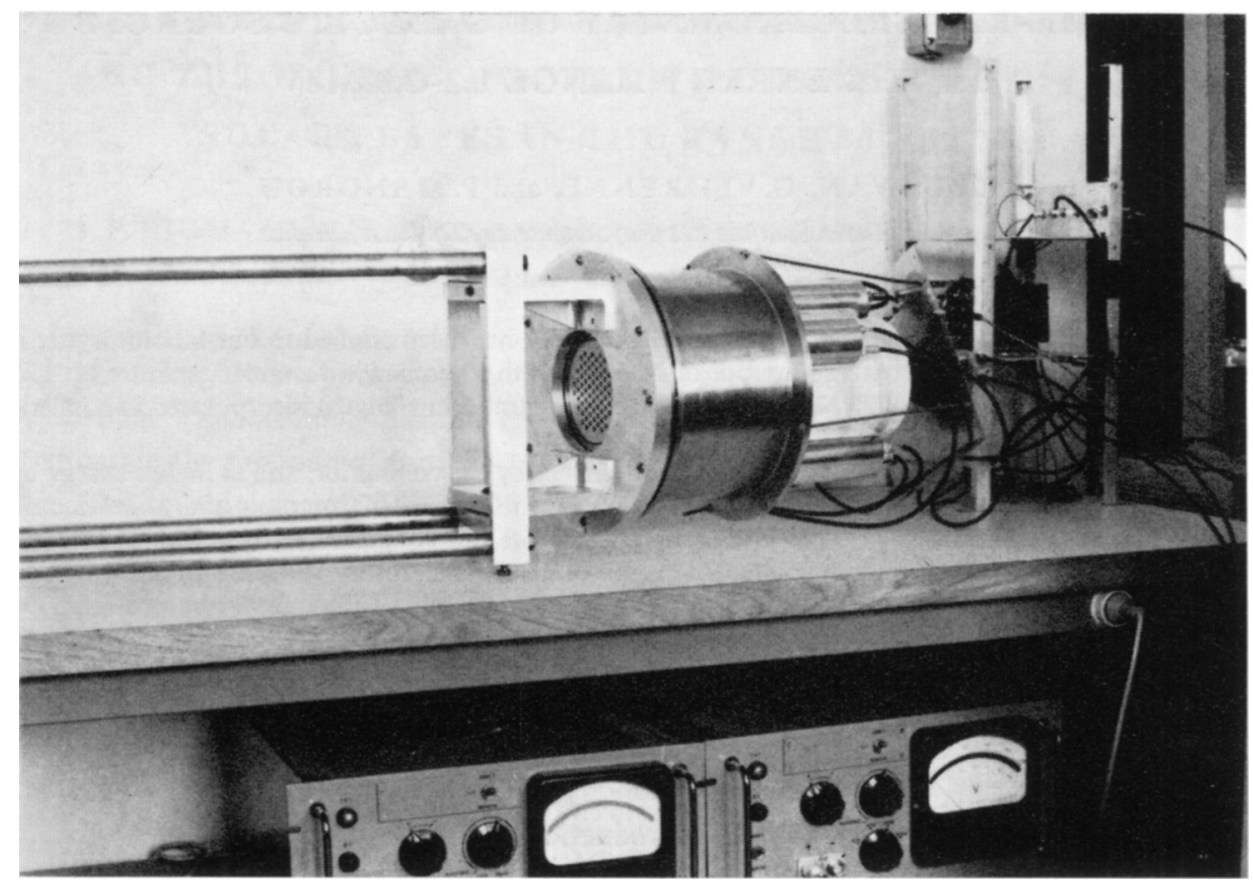

Fig. 1. Active honeycomb collimator assembly.

very high if the geometric aperture is low. Choice of the collimator thickness is made in terms of the transmission $T$ of gamma-ray flux expected in the normal direction. For a $15 \mathrm{~cm}$ high cylinder (Figure 1) it is: $T=6 \times 10^{-3}$ at $E_{\gamma}=0.511 \mathrm{MeV}$; and $T=1.5 \times 10^{-1}$ at $E_{\gamma}=3 \mathrm{MeV}$. If angular resolution is defined at half-transmission, then for different incidence energies the real aperture corresponding to a geometric diameter of holes $0.5 \mathrm{~cm}$ is as shown in Table I.

TABLE I

$\begin{array}{lllll}\text { Energy }(\mathrm{MeV}) & 0.511 & 1 & 2 & 3 \\ \text { Angular resolution } \Delta \theta & 4^{\circ} .3 & 5^{\circ} .5 & 5^{\circ} .8 & 6^{\circ} .5\end{array}$

In fact this calculated angular resolution is less than the one actually observed following a partial rejection of the incident photons which have interacted in the active collimator. This is particularly true when the energy deposited by electrons associated with a Compton scattering is not sufficient to trigger off the anticoincidence circuit associated with the collimator.

Thanks to the theoretical calculations based on the Monte Carlo Method, the real aperture can be approached by taking into account all the effects. Thus optimization can be reached when the programs are efficient. 


\section{B. DIRECTIVE DETECTOR}

It is known that the emission of electrons in the Compton and photoelectric processes becomes directive when incident energies of gamma rays increase (Davisson and Evans, 1952) (Figure 2). The distribution of the bipartition angles, defined as the angle

TABLE II

\begin{tabular}{lll} 
Energy $(\mathrm{MeV})$ & Compton electron emission & Photoelectron emission \\
\hline 0.511 & $8^{\circ}$ & $25^{\circ}$ \\
2.0 & $6^{\circ}$ & $15^{\circ}$ \\
3.0 & $4^{\circ}$ & $10^{\circ}$
\end{tabular}
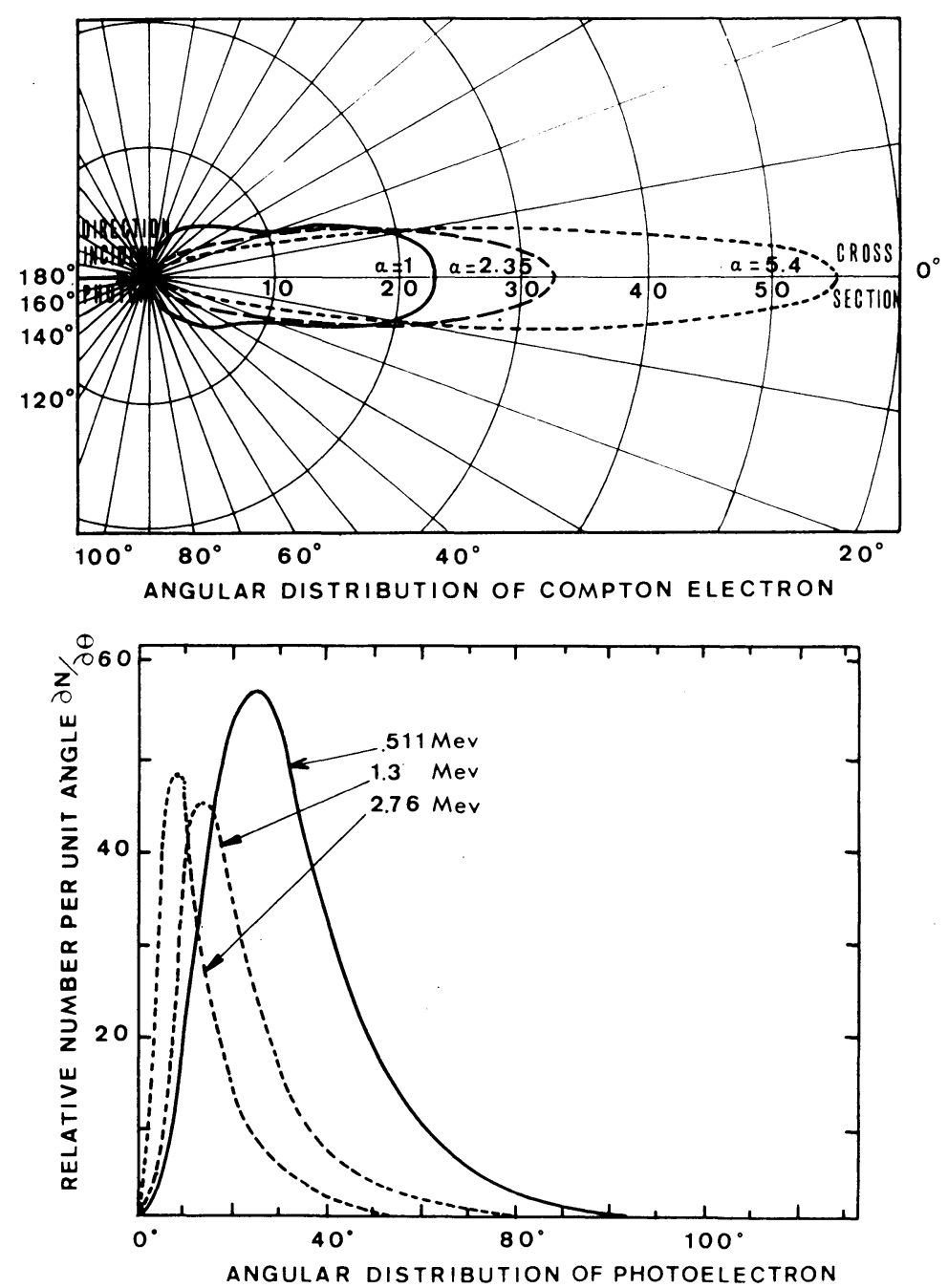

Fig. 2. Electrons emission in the processes:- Compton and photoelectric. 
in which half the particles are emitted, reflects this directivity (Table II). The latter can be restituted if the diameter of the detectors is equivalent to the electron range (Table III).

Thus for high incidence photons the electrons leave the scintillator and can be collected by a surrounded plastic which is in anticoincidence.

TABLE III

Maximum range of electrons in $\mathrm{Na} I$

$\begin{array}{ll}\text { Energy }(\mathrm{MeV}) & \text { Range }(\mathrm{cm}) \\ 1 & 0.18 \\ 2 & 0.39 \\ 3 & 0.58\end{array}$

TABLE IV

Photons incidence above which electrons can leave the crystal

Energy $(\mathrm{MeV}) \quad$ Compton electron Photoelectrons

$2 \quad 40^{\circ}(1.77 \mathrm{MeV}) \quad 18.5$

$3 \quad 22(2.76 \mathrm{MeV}) \quad 16.5$

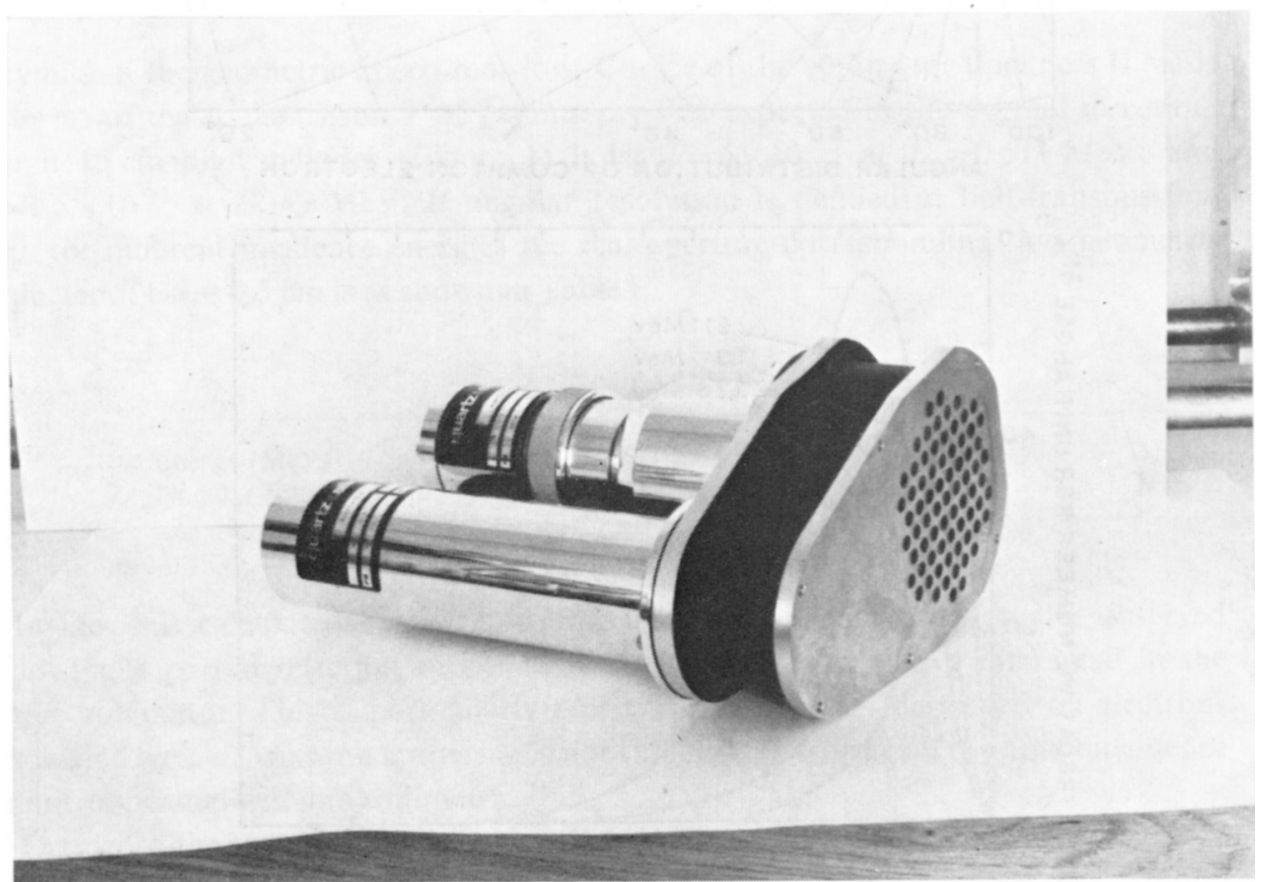

Fig. 3. Directive detector assembly. 
The chosen detector consists of $73 \mathrm{Na}$ s sticks embedded in a plastic gabarit. The dimensions of each stick are $5 \times 0.5 \mathrm{~cm}$ (Figure 3 ). A rough estimate can determine the incidence of photons above which electrons leave the crystal (Table IV).

Moreover areas of all the sticks are $20 \%$ of the total area defined in the case of an empty detector. Consequently the proper background of the detector is considerably reduced.

\section{Results and Discussions}

For instrumental calibration, a test bench has been set up at the Laboratory. Mounted by 3 rigid plates $3 \mathrm{~m}$ apart, it includes a mobile rectified plate which holds the experiment. Angular reading is made by two verniers successively providing the degree and the arcminute (Figure 4).

The radioactive sources employed are placed $3 \mathrm{~m}$ away from the detector, thereby defining an irradiation cone of 5 arc min.

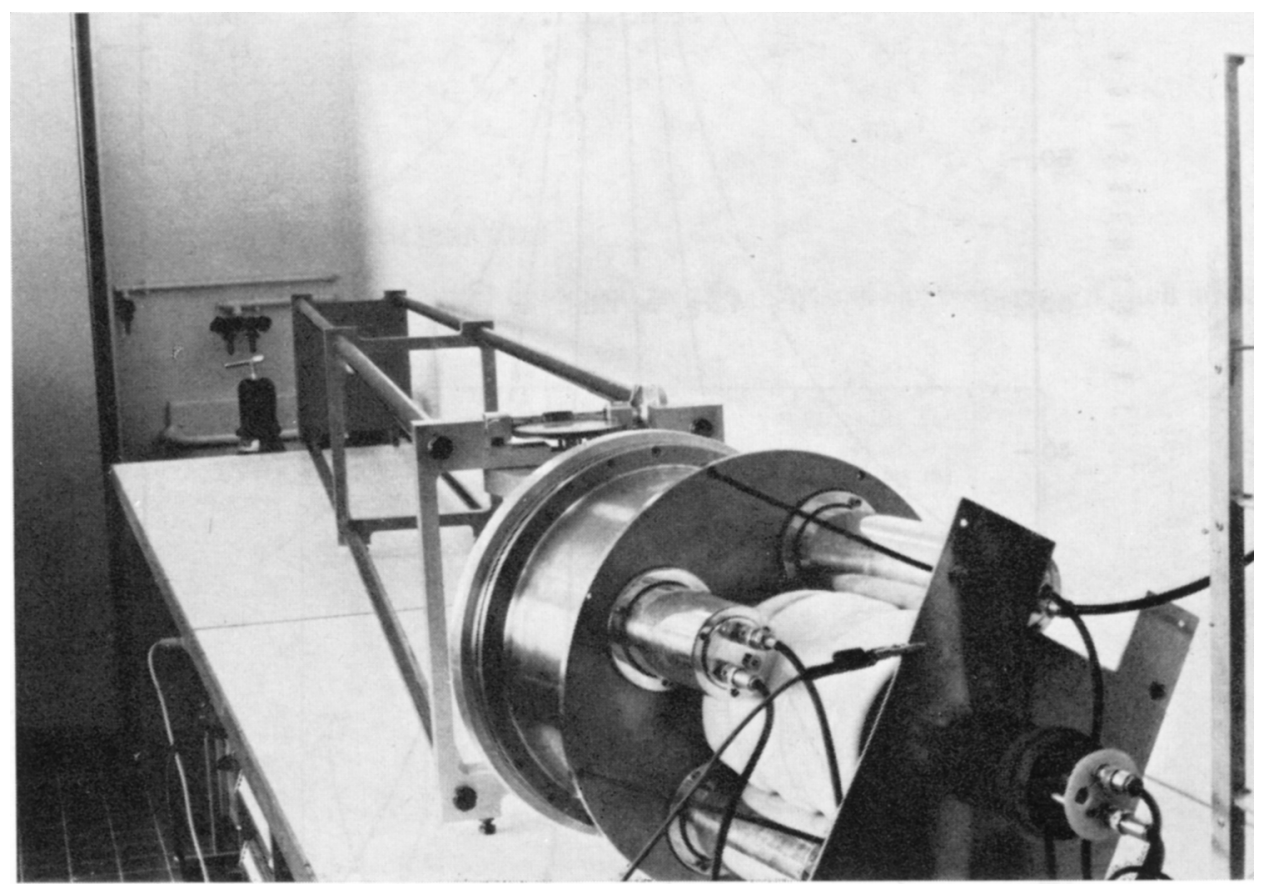

Fig. 4. Testing bench.

\section{A. COLLIMATOR CALIBRATION}

To detect all the photons transmitted through the collimator, a 1 in. $\times 4$ in. $\mathrm{Na} \mathrm{I}(\mathrm{Tl})$ crystal response is placed behind this one and is analysed in anticoincidence with the collimator.

Determination of the incident photon number is obtained by integration of the 


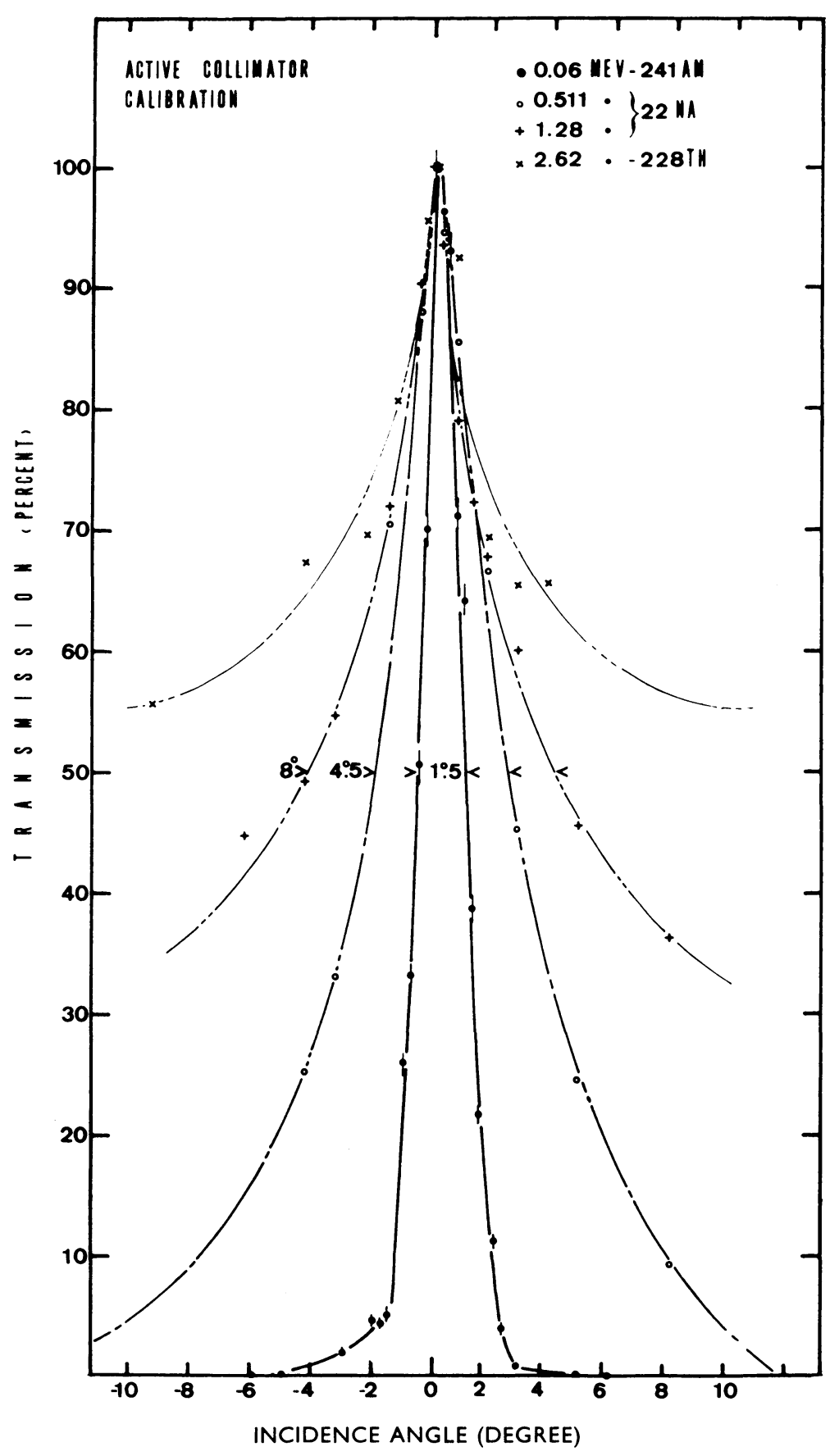

Fig. 5. Transmission of the active honeycomb collimator. 
photopeak. Because radioactive sources are not monoenergetic, the Compton spectra are mixed and make them difficult to be exploited. However the Compton spectra will be taken into consideration by using monoenergetic sources, because in this energy range this predominant contribution must be accounted for. Nevertheless this preliminary study can provide the directivity performance of the system (Figure 5).

The results obtained are reported in Table V. However for energies greater than $1 \mathrm{MeV}$ the angular resolution is difficult to define because it is over $5^{\circ}$ and the photons coming directly from the source can reach the detector. Nevertheless this effect can be reduced by coupling the collimator with the directive detector and by surrounding the unit with an active shielding.

\section{TABLE V}

$\begin{array}{ll}\text { Incident energy }(\mathrm{MeV}) & \text { Angular resolution } \\ & \\ 0.06 & 1^{\circ} .5 \\ 0.511 & 4^{\circ} .5 \\ 1.28 & 8^{\circ}\end{array}$

\section{B. DIRECTIVE DETECTOR CALIBRATION}

Before analysing the pulse-height delivered by the detector, the response of each stick,

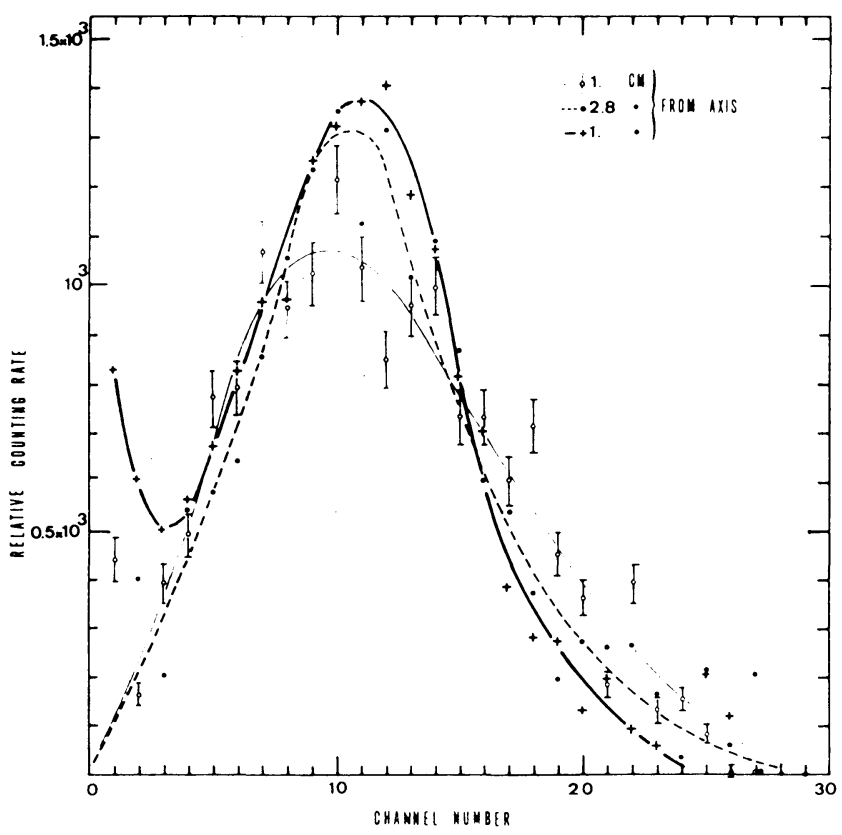

Fig. 6. Pulse-height response of detector sticks. 


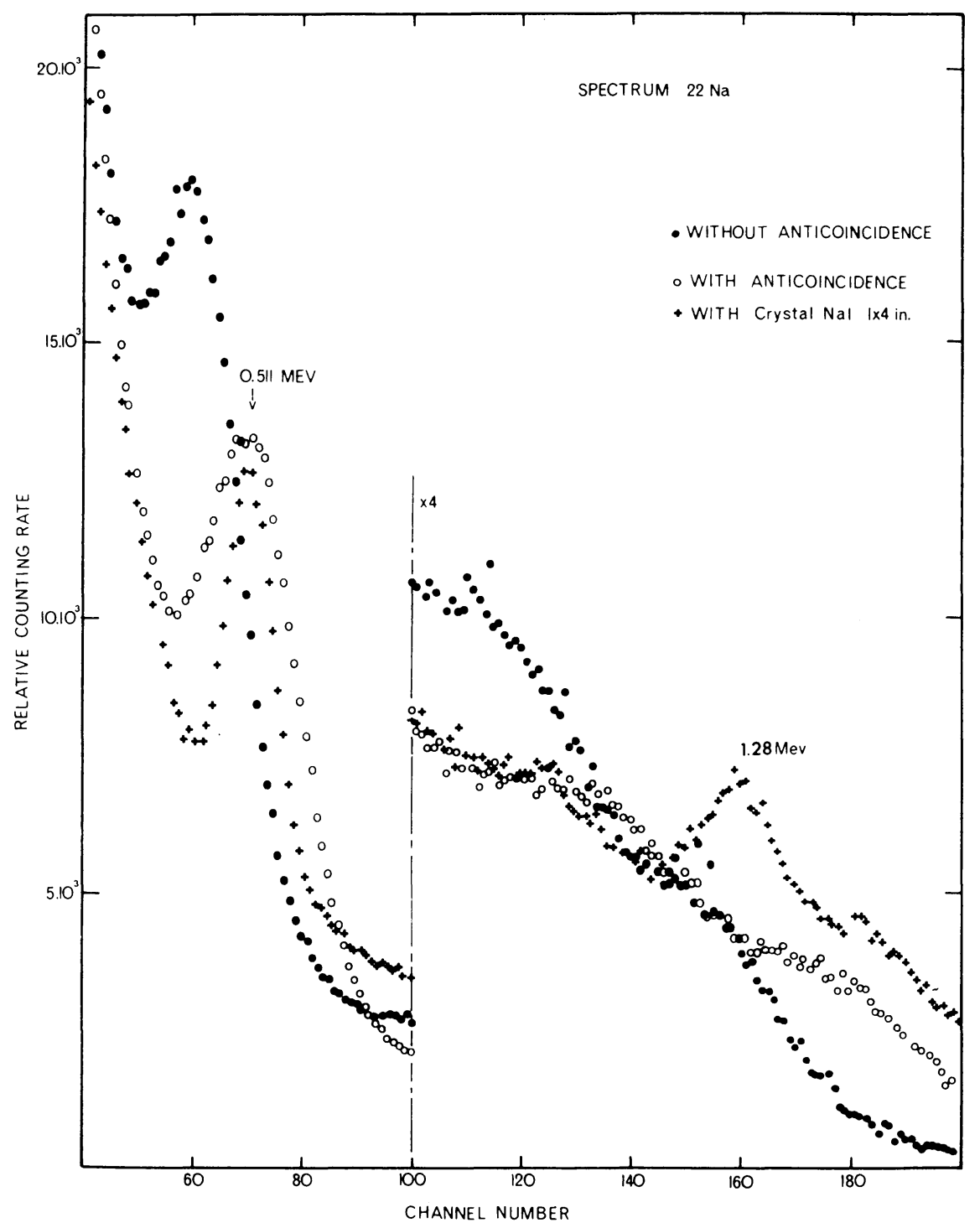

Fig. 7. Energy resolution of the detector compares with standard crystal.

which must be as identical as possible, is controlled. A weak energy gamma-ray radioactive source $\left(\mathrm{Am}^{241}\right)$ is used. Adjustment of the source in front of a stick is set up with a passive shielding. For sticks far from the axis of the photocathode a slight shift exists in the response (about $15 \%$ ) (Figure 6). The energy resolution (R) of the system by comparison with that of the standard detector reveals a slight degradation (Figure 7). Indeed, at $0.511 \mathrm{MeV}$, for instance, we obtain: 


$$
\begin{array}{ll}
\text { directive detector } & \mathrm{R}=14 \% \\
\text { standard scintillator } & \mathrm{R}=10 \% \\
(1 \text { in. } \times 4 \text { in. }) &
\end{array}
$$

The angular response of the detector is studied versus the incidence angle referred to the detector axis. Preliminary remarks have shown that Compton effect is more directive than photoelectric effect when gamma-ray energies increase. However the spectra observed at the $1.28 \mathrm{MeV}$ energy reveal an important but not complete decrease of the Compton electron distribution for high incidence angles, which means that all the electrons are not completely rejected. The contribution of this effect is difficult to estimate because we use $\mathrm{a}^{22} \mathrm{Na}$ source for this calibration and the $0.511 \mathrm{MeV}$ photopeak is juxtaposed with the Compton edge. Therefore the angular resolution of

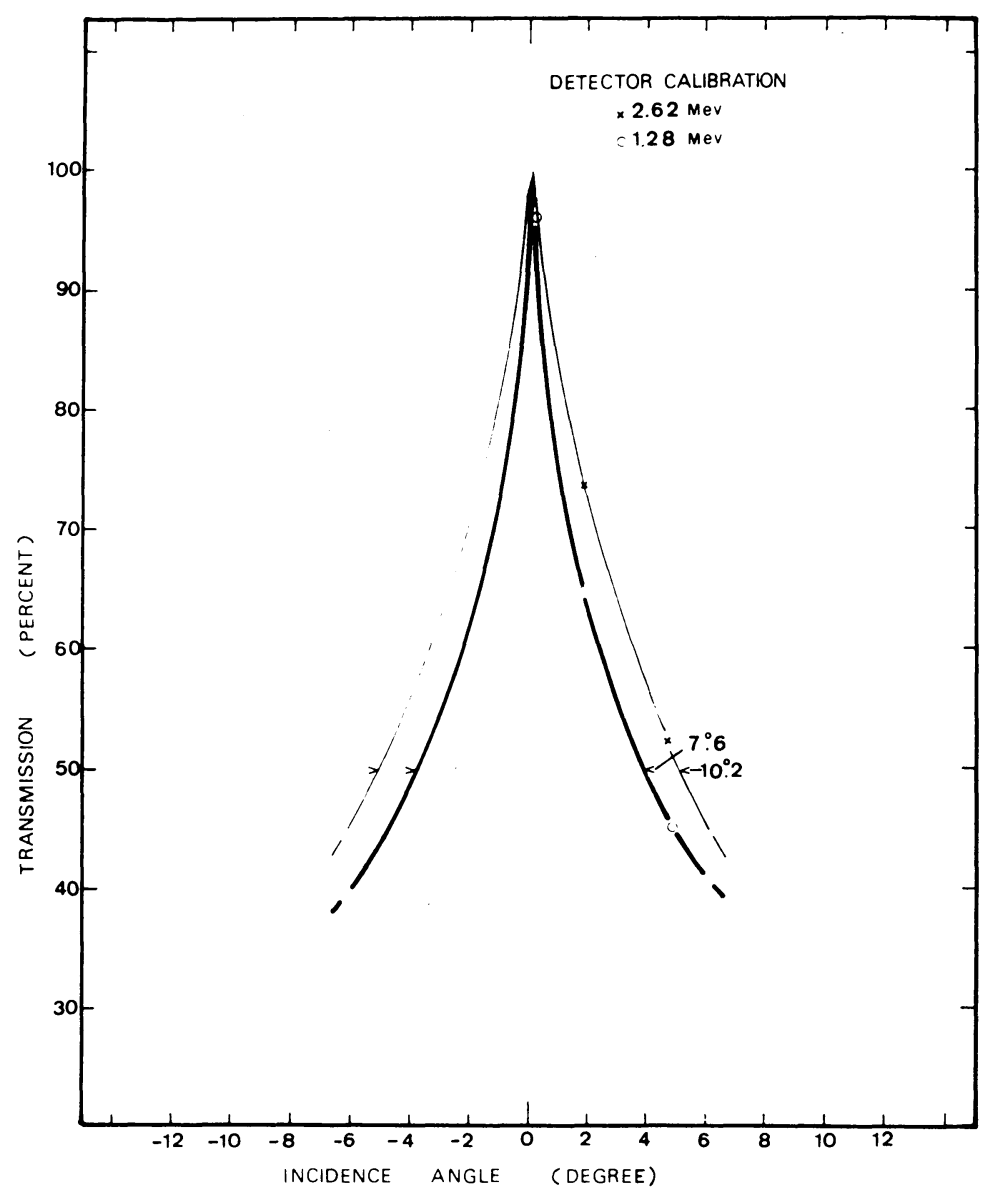

Fig. 8. Detection efficiency of INa sticks assembly. 
the detector is obtained by taking into account only the integration of the photopeak (Figure 8) (Table VI).

TABLE VI

\begin{tabular}{lc} 
Incident energy $(\mathrm{MeV})$ & Angular resolution \\
\hline 1.28 & $7^{\circ} .6$ \\
2.62 & $10^{\circ} .2$
\end{tabular}

\section{Conclusion}

Finally the product of both transmissions supplies the transmission of the detector assembly (Figure 9). Results are grouped in the Table VII (Figure 10).

These preliminary results make the observations of weak gamma-ray sources possible by reducing the background. However these first determinations of the product of collimator and detector transmissions are pessimistic. Indeed the calibration with the collimator and detector assembly have provided better results. This difference can be explained by the presence of the collimator which rejects the high incidence photons thereby limiting the diffusion effects in the detector.

The set is expected to work up to $3 \mathrm{MeV}$. For higher energy the pair production effect followed by multiple scatterings may reduce the directivity of the electron emission.

TABLE VII

$\begin{array}{llc}\text { Incident energy }(\mathrm{MeV}) & \text { Angular resolution } & \text { Lateral transmission }(\%) \\ & & \\ 0.06 & 1 \% .5 & 5 \\ 0.511 & 3 & 10 \\ 1.28 & 4.5 & 15 \\ 2.62 & 4.5 & \end{array}$

In conclusion these calibrations enable the detector shielding against photons in lateral and backward directions to be foreseen. For a residual atmospheric depth of $3.5 \mathrm{mb}$, the calculated flux of the gamma-ray background transmitted through the collimator and an active shielding with the following dimensions: wall thickness: $4 \mathrm{~cm}$; bottom thickness: $6 \mathrm{~cm}$; inside diameter: $11 \mathrm{~cm}$; is $\phi_{B}=0.668 \mathrm{c} / \mathrm{s}$ at $1 \mathrm{MeV}$ with a channel width $0.2 \mathrm{MeV}$. The signal expected from the Crab Nebula with the detector described is : $\phi_{s}=0.017 \mathrm{c} / \mathrm{s}$. Then for this source the minimum exposure time required for a counting rate over the background with $2 \sigma$ of confidence is estimated to be $2^{\mathrm{h}} 30^{\mathrm{m}}$ for $1 \mathrm{MeV}$ photon energy. 


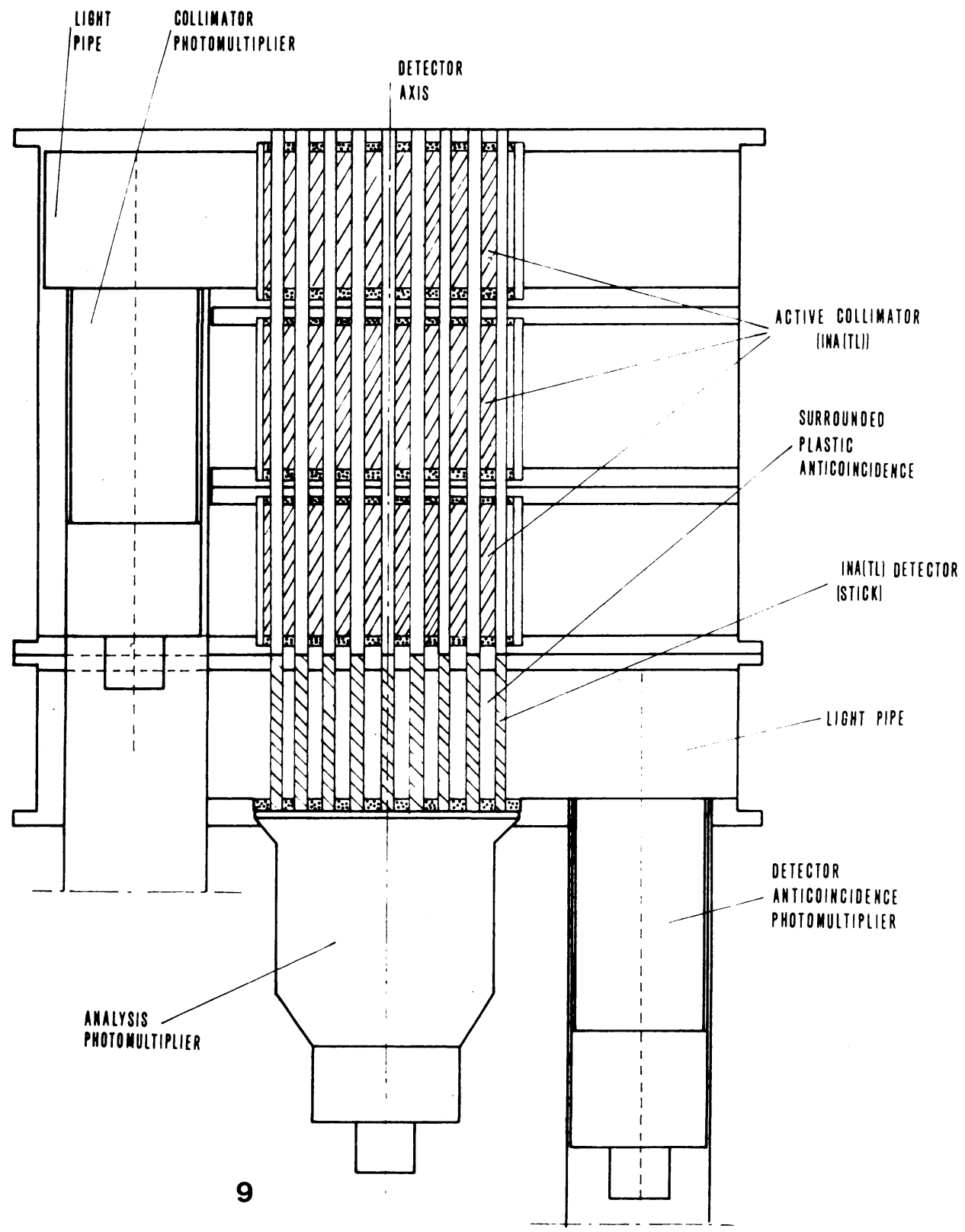

Fig. 9. Detector assembly. 


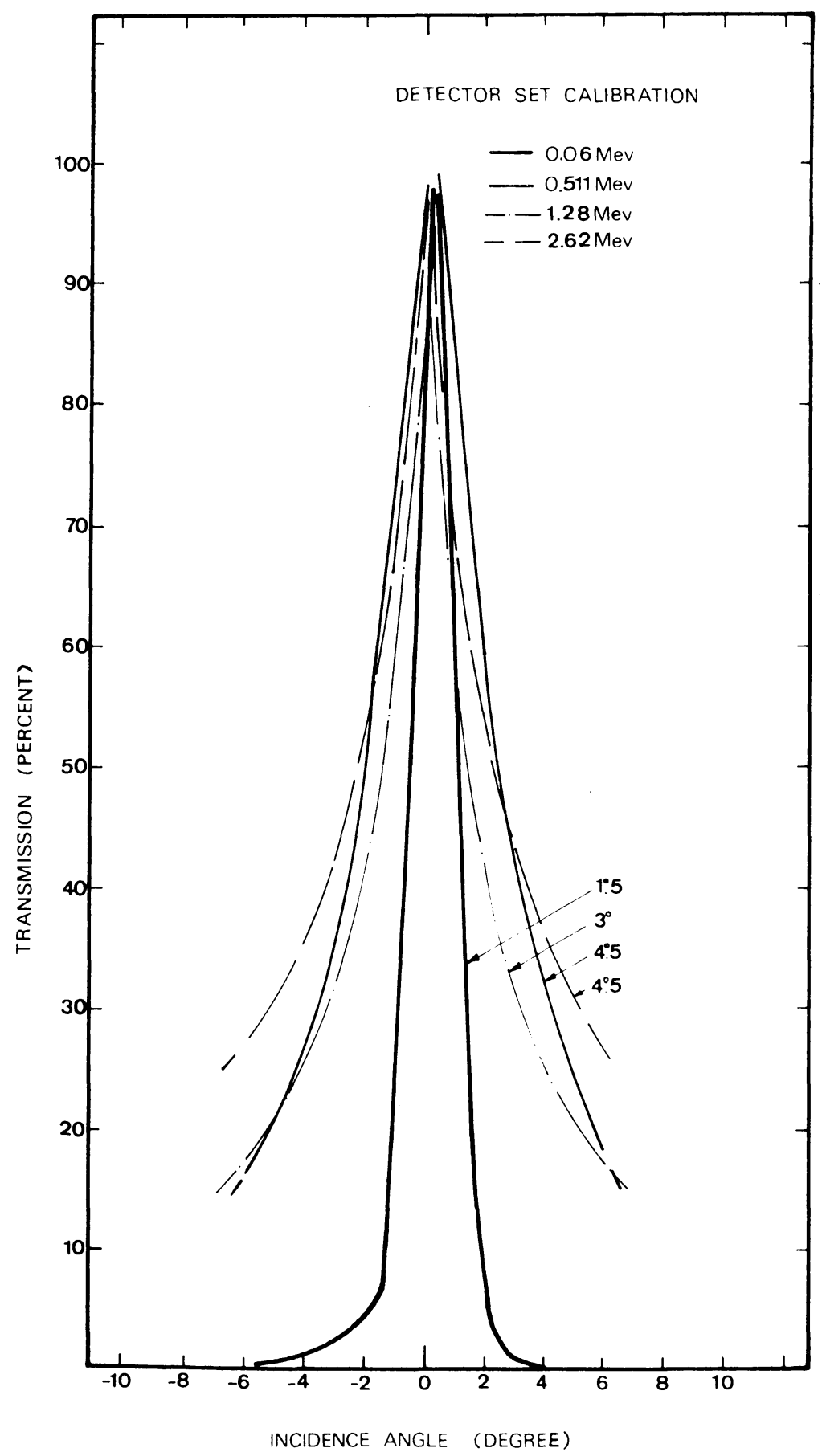

Fig. 10. Global transmission of the detector assembly. 


\section{References}

Cline, T. L.: 1961, Phys. Rev. Letters 7, 109.

Davisson, C. M. and Evans, R. D.: 1952, Rev. Method. Phys. 24, 79.

Metzger, A. E., Anderson, E. C., Van Dilla, M. A., and Arnold, J. R.: 1964, Nature 204, 766.

Peterson, Laurence E., Jacobson, Allan S., Pelling, R. M., and Schwartz, Daniel A.: 1968, Can J. Phys. 46, 10. 